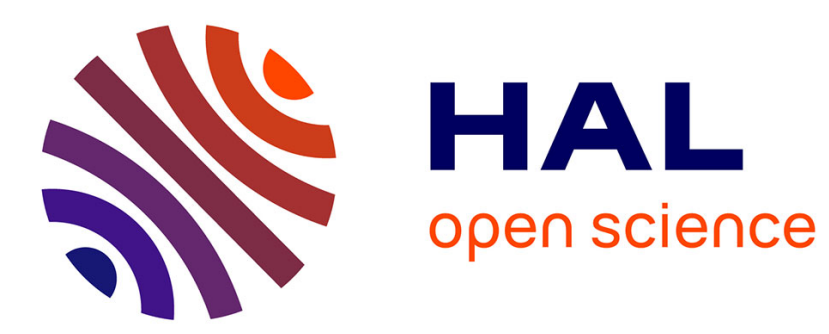

\title{
Excitons in a single quantum well wire: optical response and application to the gratings
}

\author{
A. d'Andrea, L. Pilozzi
}

\section{To cite this version:}

A. d'Andrea, L. Pilozzi. Excitons in a single quantum well wire: optical response and application to the gratings. Journal de Physique IV Proceedings, 1993, 03 (C5), pp.C5-385-C5-388. 10.1051/jp4:1993581 . jpa-00251668

\section{HAL Id: jpa-00251668 https://hal.science/jpa-00251668}

Submitted on 1 Jan 1993

HAL is a multi-disciplinary open access archive for the deposit and dissemination of scientific research documents, whether they are published or not. The documents may come from teaching and research institutions in France or abroad, or from public or private research centers.
L'archive ouverte pluridisciplinaire HAL, est destinée au dépôt et à la diffusion de documents scientifiques de niveau recherche, publiés ou non, émanant des établissements d'enseignement et de recherche français ou étrangers, des laboratoires publics ou privés. 


\title{
Excitons in a single quantum well wire: optical response and application to the gratings
}

\author{
A. D'ANDREA and L. PILOZZI* \\ Istituto di Metodologie Avanzate Inorganiche, CNR, 00016 Monterotondo S., Roma, Italy \\ * Dipartimento di Fisica, II Università di Roma, via E. Carnevale, 00173 Roma, Italy
}

\begin{abstract}
Variational envelope function of an exciton in a single quantum well wire (SQWW) is described in the framework of the effective mass approximation for two-band model. The SQWW considered has thickness values : $\mathrm{L}_{\mathrm{z}}<\mathrm{a}_{\mathrm{B}}$, along the growth axis of the sample, and $\mathrm{L}_{\mathrm{x}}>$ $3 a_{B}$ for the lateral dimension (where $a_{B}$ is the Bohr radius). Exciton polarizations and optical response for SQWW and for a grating of QWWs are given. The role of the local fields on the optical response of these two different superstructures is pointed out.
\end{abstract}

1.Introduction: The improvement in microstructure fabrication allows to study the exciton behaviour in single quantum well wires (SQWWs) as a function of lateral dimension. The techniques widely used to define QWWs (electron-beam lithography or optical holography followed by dry etching) give 1D-structures that show, along the lateral dimension, perfect confinement of the exciton and large dead-layer effect ${ }^{1}$ ). In the present paper we study the local field effects due to the grating periodicity in rather large QWWs. This study is performed comparing optical response in a SQWW and in a grating of QWWs. In Section 2 the exciton properties in a SQWW of GaAS/GaAlAs are discussed in the framework of two band model and perfect confinement of the exciton. In Section 3 optical response of a single QWW and of a grating of QWW are computed and the local field effects for these two superstructures are briefly discussed. Conclusions are given in Section 4.

2.Exciton properties in a SQWW: Let us consider a SQWW where $y$ is the axis of symmetry of the wire and $L_{z}<a_{B}$ is the dimension along the growth axis, while $L_{x}>3 a_{B}$ is the lateral dimension, and $a_{B}$ is the Bohr radius.

Modelling the Wannier exciton as perfectly confined along $x$-axis, and neglecting the contribution of the center-of-mass motion along $\mathrm{y}$-axis, the exciton Hamiltonian (with $\mathrm{h}=1$ ) is:

$$
\mathrm{H}_{\mathrm{ex}}=-\frac{1}{2 \mathrm{M}}\left[\frac{\partial 2}{\partial \mathrm{X}^{2}}+\frac{\partial^{2}}{\partial \mathrm{Z}^{2}}\right]-\frac{\nabla_{\mathrm{r}^{2}}}{2 \mu}-\frac{1}{\varepsilon_{\mathrm{o}} \mathrm{r}}+\mathrm{V}_{\mathrm{e}}\left(\mathrm{z}_{\mathrm{e}}\right)+\mathrm{V}_{\mathrm{h}}\left(\mathrm{z}_{\mathrm{h}}\right)
$$

where $\mu$ and $M$ are respectively the reduced and the total exciton masses and $V_{e}\left(z_{e}\right)$ and $V_{h}\left(z_{h}\right)$ are the confinement potentials for electron and hole, along $z$-axis. A well suited trial envelope function for an exciton in a SQWW is:

$$
\phi_{e x}(r, X, Z)=N_{m n !} Q_{m}(x, X) R_{n}\left(z_{e}, z_{h}\right) \phi_{l}(r)
$$

where $r=r_{e^{-}} r_{h}$ is the relative vector distance between $e-h$ and $(X, Y, Z)$ are the coordinates of the centerof-mass. $\mathrm{N}_{\mathrm{mnl}}$ is the normalization constant in the $(r, X, Z)$ space, $\phi_{1}(\mathbf{r})$ is the hydrogenic wave function (for $l=1$ is: $\exp [-\alpha r]), Q_{m}(x, X)$ is the D'Andrea-Del Sole $\left(^{2}\right)$ confinement function $\left(L_{X}>3 a_{B}\right.$ ), and $R_{n}\left(z_{e}, z_{h}\right)$ is a product of two subbands respectively for electron and hole.

In order to minimize the first momentum of the total Hamiltonian of eq.(1), as a function of two variational parameters, namely: $1 / \alpha$ the exciton radius embodied into the hydrogenic wave function 
$\phi_{1}(r ; \alpha)$, and 1/P the exciton dead-layer that appears in the confinement function $Q_{m}(x, X ; P)(2)$, we proceed in two steps.

The first step consists in the minimization of the Hamiltonian matrix element,

$$
\begin{aligned}
& <\phi_{\text {ex }}\left|H_{\text {ex }}{ }^{0}\right| \phi_{e x}>= \\
& =N_{\left.m n\right|^{2}}<Q_{m}(x, X ; P) \phi_{I}(r ; \alpha)\left|W(z)\left[-\frac{1}{2 M} \frac{\partial 2}{\partial X^{2}}-\frac{\nabla_{r}{ }^{2}}{2 \mu}-\frac{1}{\varepsilon_{0} r}\right]\right| Q_{m}(x, X ; P) \phi_{1}(r ; \alpha)> \\
& =\text { minimum . }
\end{aligned}
$$

as a function of variational parameter $P$ taking $\alpha=1 / a_{B}=$ constant . Note that the integrand is very similar to that used in the single quantum well (SQW) ${ }^{3}$ ) except for the weight-function $W(z)=\int d Z\left|R_{n}(z, Z)\right|^{2}$ not present in that case. Moreover, integrating the matrix element of eq.(3) on the z-relative coordinate, we obtain a $2 \mathrm{D}$-averaged Coulomb potential $(4)$.

The second step consists in the minimization of the total exciton Hamiltonian as function of $\alpha$ parameter (for $\mathrm{P}=$ constant ),

$$
<\phi_{e x}\left|H_{e x}\right| \phi_{e x}>=<\phi_{e x}\left|H_{e x}{ }^{0}\right| \phi_{e x}>-\frac{1}{\mu} N_{\left.m n\right|^{2}}<R_{n}(z, Z) \phi_{I}(r ; \alpha)|w(x, P)| \frac{\partial R_{n}}{\partial z} \frac{\partial \phi_{I}}{\partial z}>,
$$

where $w(x, P)$ is $w(x, P)=\int d X\left|Q_{m}(x, X ; P)\right|^{2}$.

The general minimum is obtained by a self-consistent procedure. The convergence of the process is usually very fast and the minimum is reached after a few cycles of computation.

The exciton dipole moment in SQWW per unit of length is:

$$
f_{o} / L_{y}=\mu_{c v} N_{m n l}\left|\int d X d Z Q_{m}(x=0, X) R_{n}(z=0, Z) \phi_{\mid}(r=0)\right| .
$$

The physical parameters used for the calculations in $\mathrm{GaAs} / \mathrm{GaAlAs}$ system are: $\varepsilon_{\mathrm{o}}=12.6, \mathrm{M}=0.3 \mathrm{~m}$, Ryd. $=4.2 \mathrm{meV}, 4 \pi \alpha=0.0022$ and $E_{T O}=1.515 \mathrm{eV}$. In fig. 1 is shown the exciton dipole moment $f_{\alpha} / L_{y}$ for all the range of $L_{z}$ and for two different values of the lateral dimension $L_{X}(2)$. For large value range of $L_{z}\left(L_{z}>a_{B}\right)$ we use D'Andrea-Del Sole confinement funtion $(3)$ also for $z$-motion of exciton.

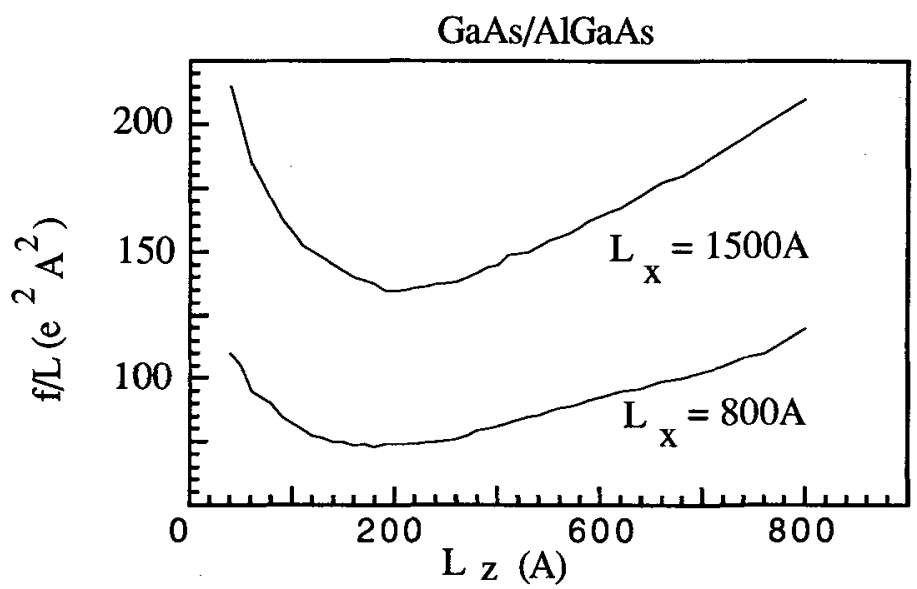

Fig.1 Exciton dipole moment per unit of length in a single quantum well wire.

3.Optical Response: Now, let us consider a SWW embedded in a semi-infinite solid with the surface in the plane $(x, y)$ and with the static dielectric constant $\varepsilon_{0}$. Taking the incident electric field with the momentum $K=\left(K_{x}, 0, K_{z}\right)$, the Maxwell equations decouple in $S$ and $P$ polarizations and it can be exactly solved, see Refs. $(2,5)$. We will neglect the stationary polariton and consider only the optical 
response of the resonant modes $\left(^{6}\right)$. The Fourier transformed polarizability for SQWW (for $n=0$ and $l=1)$ is:

$$
\begin{aligned}
& 4 \pi P\left(k_{x}, Z\right)=\left(\varepsilon_{0}-1\right) E\left(k_{x}, Z\right)+ \\
& \quad+\sum_{m} N_{m}{ }^{2} \frac{S_{0}}{E_{m^{-}} h \omega} Q_{m}^{*}\left(-k_{x}\right) R^{*}(Z) \int d k_{x}^{\prime} Q_{m}\left(-k_{x}^{\prime}\right) \int d Z^{\prime} R\left(Z^{\prime}\right) E\left(k_{x}^{\prime}, Z^{\prime}\right),
\end{aligned}
$$

where $S_{0}$ embodies the valence-conduction dipole moment transition.

Let us consider a $S Q W W$ of dimensions $L_{x}=1500 A$ and $L_{z}=100 A$. Note that we will use in the calculation an unrealistically low $\Gamma-$ value $(=0.03 \mathrm{meV})$ in order to point out rather small effects in the theoretical spectra .

The reflectivity for $S$-polarized light is shown in fig. 2 a for incident angle: $\phi_{1}=30^{\circ}$. This result is obtained normalizing the data in a small angle around the reflection condition. The two peaks are due to the exciton center-of-mass quantization $(n=1$ and $n=3)$ along the $x$-axis in the wire . In fig. $2 b$ we compute for the same system the reflectivity with reflection angle $\phi_{r}$ different from the incident one, namely: $\omega / c \sin \left(\phi_{\mathrm{r}}\right)=\omega / \mathrm{c} \sin \left(\phi_{\mathrm{l}}\right) \pm 2 \pi /\left(10 \mathrm{~L}_{\mathrm{x}}\right)$. The intensity of the peaks decreases respect to the reflection condition $\phi_{r}=\phi_{l}$, in fact, the term $2 \pi /\left(10 \mathrm{~L}_{\mathrm{X}}\right)$ is of the same order of magnitude than the light impulse. Moreover, reflection peaks at exciton energies $n=1,2$ for $q_{x} \pm 2 \pi /\left(10 L_{x}\right)$ shows different intensity values (see fig. $2 b$ where $R_{+}$is the solid line, $R_{-}$dashed line and $R_{+}>R_{-}$).

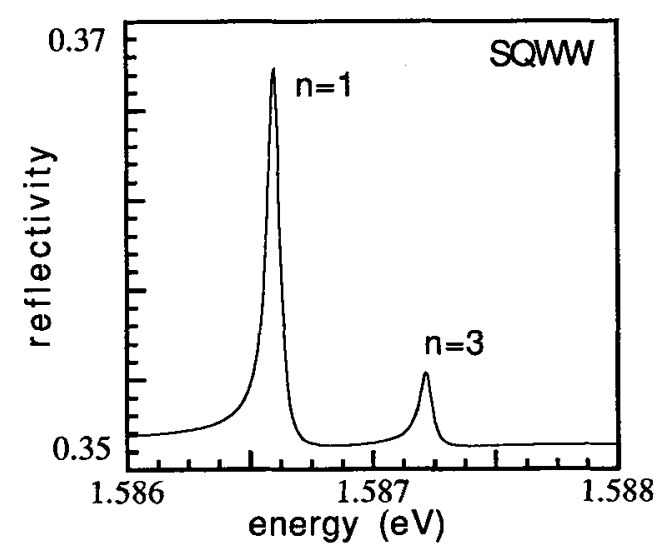

Fig.2a- Reflectivity for SQWW $\left(\phi_{r}=\phi_{1}\right)$

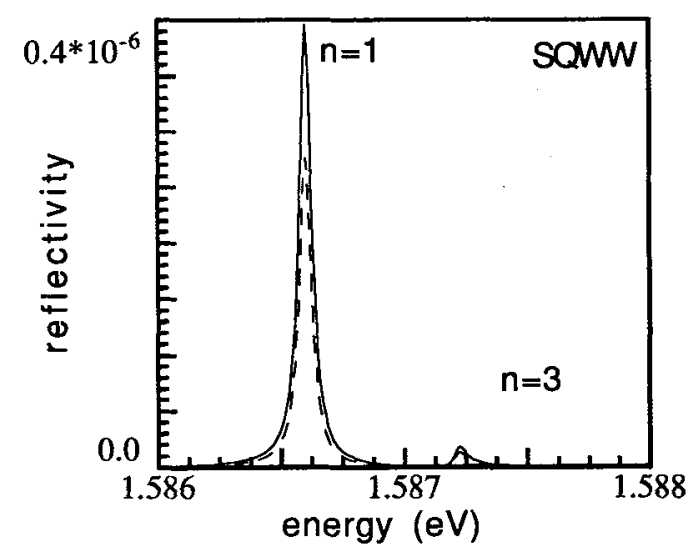

Fig.2b- Reflectivity for SQWW $\left(\phi_{r} \neq \phi_{l}\right)$ Now, let us consider a grating of QWWs embedded in a semi-infinite solid of dielectric constant $\varepsilon_{0}$ (2). The exciton polarizability is,

$$
\begin{aligned}
& 4 \pi P\left(k_{x}, Z\right)=\left(\varepsilon_{o}-1\right) E\left(k_{x}, Z\right)+ \\
& +\frac{1}{d} \sum_{m} N_{m}^{2} \frac{S_{0}}{E_{m^{-}} h \omega} Q_{m}^{*}\left(-k_{x}\right) R^{*}(Z) \sum_{G} Q_{m}\left(-k_{x}+G\right) \int d Z^{\prime} R\left(Z^{\prime}\right) E\left(k_{x}+G, Z^{\prime}\right),(7)
\end{aligned}
$$

where $d$ is the distance between adjacent $Q W W s$ and $G=2 \pi / d$ is the reciprocal lattice vector.

In fig. $3 \mathrm{a}$ is shown the reflectivity $(\mathrm{G}=0)$ for $\mathrm{q}_{\mathrm{x}}=\omega / \mathrm{c} \sin \left(\phi_{\mathrm{l}}\right)$, where $\phi_{\mathrm{l}}=30^{\circ}$ is the incident angle, and for two different distances between adjacent wires, namely : $d=5000 \mathrm{~A}$ and $\mathrm{d}=1600 \mathrm{~A}$. Obviously, in the limit $\mathrm{d}->\infty$ the intensity of the peaks decreases and it could converge to the static bulk reflectivity for a single spot $G$ and to the SQWW reflectivity of fig.2a if integrated on the choosen small angle. In fig. $3 b$ is computed the reflectivity for impulse parallel to the surface respectively: $q_{x}=\omega / c \sin \left(\phi_{l}\right) \pm G$. Note that in this case we obtain a response qualitatively different from the case $G=0$, in fact, due to 
different selection rules for reflection and out of reflection cases, also odd states $(n=2)$ appears in the spectra. Moreover, for asymmetric conditions $\left(\phi_{1} \neq 0\right)$ there is a strong redistribution of the intensities among the exciton peaks $(n=1,2,3)$ and reflectivities are: $R_{+}<R$.

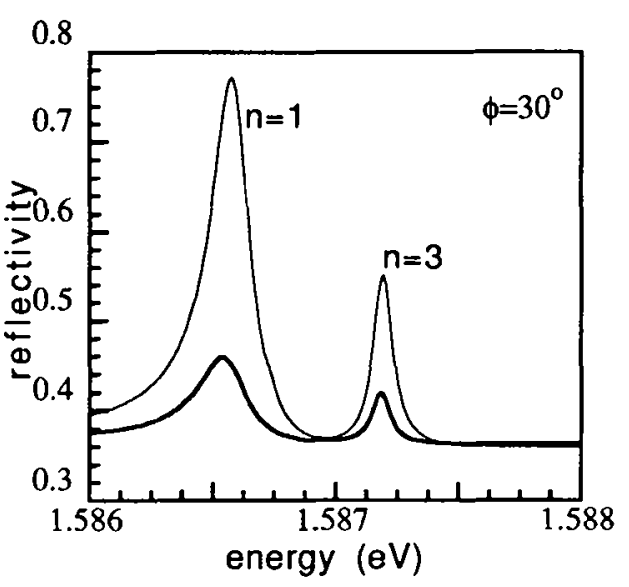

Fig.3a - Reflectivities of a grating of QWWs for $\mathrm{G}=0$ and $\mathrm{d}$ : $1600 \mathrm{~A}$ (thin solid line) and $5000 \mathrm{~A}$ (thick solid line).

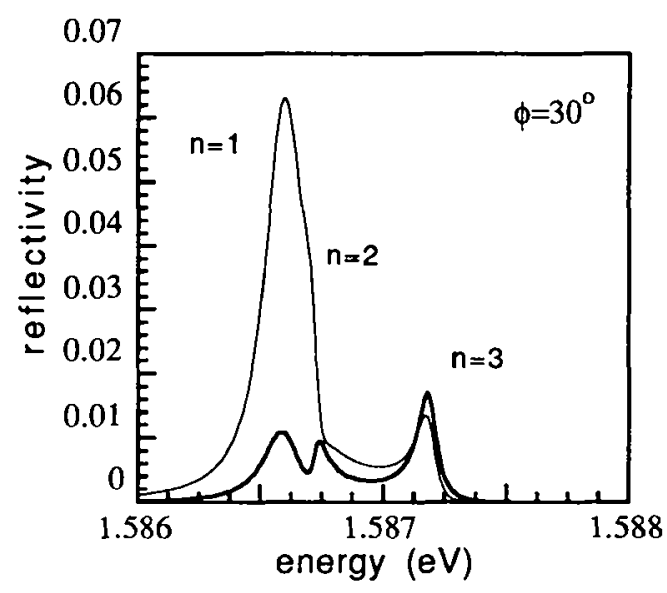

Fig.3b - Reflectivities of a grating of QWWs for $\mathrm{G}=+2 \pi / \mathrm{d}$ (thick solid line) and for $\mathrm{G}=-2 \pi / \mathrm{d}$ (thin solid line).

Quantitative comparison with the reflectivity in SQWW of fig.2a is not easy, dues to the different normalization conditions in a grating and in a single wire. The study of the reflectivities ratio $R_{+} / R_{-}$ between ligth reflected at $q_{x} \pm \mathbf{G}$ is a finger print of the local field effects . Finally, preliminary results for $Q W W s$ with rather large lateral thickness $\left(L_{x}=10 a_{B}\right)$ give non-negligible optical response for out of reflection condition in these superstructures.

4.Conclusions: In conclusion, we present a framework for computing optical response in QWWs in all the range of lateral dimensions. Preliminary results seem to allow a compared study of the local field effects on the optical response from SQWW to the periodic grating in QWWs with rather large lateral dimension.

Acknowledgments: The authors are indebited to Prof.R.Del Sole for usefull discussions and suggestions.

\section{References :}

[1] - H.Lage, D.Heitmann, R.Cingolani, P.Grabow and K.Ploog, Phys.Rev.B44(1991)6550.

[2] - A.D'andrea and R.Del Sole, Phys.Rev.B46 (1992) 2363.

[3] - A.D'Andrea and R.Del Sole,Phys.Rev.B 41 (1990) 1413.

[4] - S.Glutsch and F.Bechstedt, Phys.Rev.B 47 (1993)

[5]-E.L.Ivchenko and A.V.Kavokin, Superlattic. Microstr.v12,(1992) 317, Sov.Phys.Solid State 34,(1992) 968

[6] - F.Tassone and F.Bassani, Il Nuovo Cimento D14 (1992) 1241 